\title{
Dynamic Changes in Interneuron Morphophysiological Properties Mark the Maturation of Hippocampal Network Activity
}

\author{
Camille Allene, ${ }^{1,2,3}$ Michel A. Picardo, ${ }^{1,2,3}$ Hélène Becq, ${ }^{1,2,3}$ Goichi Miyoshi, ${ }^{4}$ Gord Fishell, ${ }^{4}$ and Rosa Cossart ${ }^{1,2,3}$ \\ ${ }^{1}$ Inserm Unité 901, 2Université de la Méditerranée, UMR S901 Aix-Marseille 2, and ${ }^{3}$ INMED, 13009 Marseille, France, and ${ }^{4}$ New York University \\ Neuroscience Institute, Departments of Physiology and Neuroscience, and Neural Science, and Langone Medical Center, New York, New York 10016
}

\begin{abstract}
During early postnatal development, neuronal networks successively produce various forms of spontaneous patterned activity that provide key signals for circuit maturation. Initially, in both rodent hippocampus and neocortex, coordinated activity emerges in the form of synchronous plateau assemblies (SPAs) that are initiated by sparse groups of gap-junction-coupled oscillating neurons. Subsequently, SPAs are replaced by synapse-driven giant depolarizing potentials (GDPs). Whether these sequential changes in mechanistically distinct network activities correlate with modifications in single-cell properties is unknown. To determine this, we studied the morphophysiological fate of single SPA cells as a function of development. We focused on CA3 GABAergic interneurons, which are centrally involved in generating GDPs in the hippocampus. As the network matures, GABAergic neurons are engaged more in GDPs and less in SPAs. Using inducible genetic fate mapping, we show that the individual involvement of GABAergic neurons in SPAs is correlated to their temporal origin. In addition, we demonstrate that the SPA-to-GDP transition is paralleled by a remarkable maturation in the morphophysiological properties of GABAergic neurons. Compared with those involved in GDPs, interneurons participating in SPAs possess immature intrinsic properties, receive synaptic inputs spanning a wide amplitude range, and display large somata as well as membrane protrusions. Thus, a developmental switch in the morphophysiological properties of GABAergic interneurons as they progress from SPAs to GDPs marks the emergence of synapse-driven network oscillations.
\end{abstract}

\section{Introduction}

As development proceeds, the changing dynamics of spontaneous coordinated neuronal activity is controlled by distinct cellular mechanisms, resulting in a stereotypic sequence being associated with the maturation of network activity patterns (Allene and Cossart, 2010; Blankenship and Feller, 2010). Interestingly, the timing and mechanisms used in the maturation of spontaneous patterned activity is remarkably robust across brain structures, indicating that the signals linking activity to the maturation of neurons and circuits could be widely conserved.

Still, it remains unknown whether the developmental sequences in network activity patterns impact the morphophysiological properties of the individual neurons. Addressing this issue is crucial for understanding the role of activity in development, as

\footnotetext{
Received Jan. 6, 2012; revised March 5, 2012; accepted March 15, 2012.

Author contributions: R.C. designed research; C.A., M.A.P., and H.B. performed research; G.M. and G.F. contributed unpublished reagents/analytic tools; C.A. analyzed data; C.A., G.M., G.F., and R.C. wrote the paper.

This work was supported by grants from the European Research Council (ERC FP7 Young Investigators \#242852), the Fondation pour la Recherche Medicale (Equipe FRM 2008), the Fondation Bettencourt Schueller, INSERM, the Ville de Marseille and Region PACA (Provence Alpes (ôte d'Azur), and the Fondation pour la Recherche sur le Cerveau. R.C. is funded by the CNRS. C.A. was funded by a fellowship from the FRM. Research in the Fishell laboratory is supported by the National Institutes of Health (NIH Grants R01MH071679 and R01NS039007), as well as generous support from New York State Stem Cell Science and the Simons Foundation. G.M. is supported by a grant from the National Alliance for Research on Schizophrenia and Depression. We thank Pr. Jane Johnston for providing the Mash $1{ }^{\text {CreERTM }}$ mouse. We thank Drs. A. Baude, S. Feldt, and V. Villette for critical comments.

Correspondence should be addressed to Rosa Cossart at the above address. E-mail: rosa.cossart@inserm.fr.

DOI:10.1523/JNEUROSCI.0081-12.2012

Copyright $\odot 2012$ the authors $\quad 0270-6474 / 12 / 326688-11 \$ 15.00 / 0$
}

it should help elucidate the influence of early correlated activities on the maturation of specific single-cell properties.

We have recently dissected the maturation of correlated activity patterns in both the rodent hippocampus and neocortex using electrophysiology and calcium imaging (Crépel et al., 2007; Allene et al., 2008). In both regions, the earliest coherent electrical patterns of activity emerge around birth in the form of synchronous plateau assemblies (SPAs), so called because of their characteristic spatial-temporal dynamics: SPAs involve discrete groups of gap-junction-coupled neurons producing correlated calcium plateaus associated with suprathreshold membrane potential oscillations. SPAs are gradually switched off toward the end of the first postnatal week in rodents, when they are replaced by synapse-driven giant depolarizing potentials (GDPs) (Ben-Ari et al., 1989) that synchronize larger neuronal populations. Here we asked whether the involvement of an individual neuron in SPAs or GDPs is mirrored by changes in their specific morphophysiological properties. We focused on GABAergic neurons for two reasons. First, GABAergic transmission is central for the generation of GDPs (Ben-Ari et al., 1989; Garaschuk et al., 1998; Sipilä et al., 2005; Bonifazi et al., 2009), suggesting that SPAs, by occurring before the onset of GDPs, could be a central step for the maturation of interneurons. Second, the GABAergic neuron population is characterized by its morphophysiological diversity. If diverse subtypes are affected by early network activities in a similar way, it would imply that the impact of correlated activity is universal and cell-type-independent. 

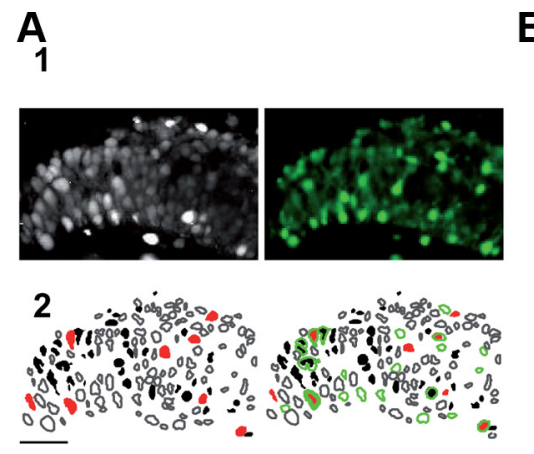

B

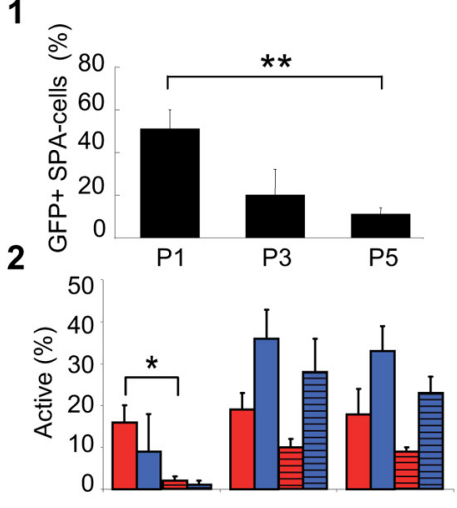

$C_{1}$

$D_{1}$
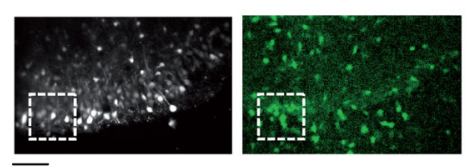

2
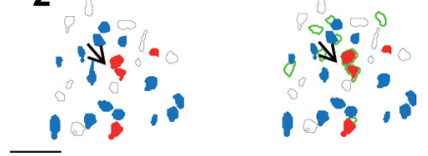

3
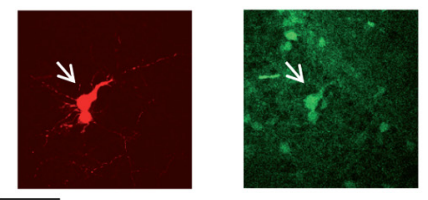

Figure 1. GABAergic neurons are preferentially involved in SPAs at early postnatal stages (P0 - P5). A1, Two-photon calcium fluorescence image from the CA3 region of a P1 GAD67-KI-GFP mouse (left) and the GFP signal from the same field (right). $\boldsymbol{A 2}$, Automatically detected contours of the cells (left) from the calcium fluorescence image (A1) and overlap of this contour map with that of GFP-positive cells (right). Open contours, Silent cells; black-filled contours, cells producing calcium spikes; red-filled contours, SPA cells; green open contours, GFP-positive cells. Note that in this case, half of the SPA cells are GFP-positive. B1, Histogram indicating the fraction of SPA cells that are GFP-positive at three time points (P1, P3, P5). B2, Histogram indicating the fraction of interneurons (solid-colored bars) and pyramidal cells (striped bars) involved in SPAs (red) or in GDPs (blue) relative to the total interneuron or pyramidal cell population, respectively, as a function of time. C1, Two-photon calcium fluorescence image from the CA3 region of a P5 GAD67-KI-GFP mouse (left) and the GFP signal from the same field (right). C2, Contour map of the imaged cells (left) from the boxed areas in C1 and superimposed on the GFP-positive cell contour map (right). Open contours, Silent cells; red-filled contours, SPA cells; blue-filled contours, GDP cells; green open contours, GFP-positive cells. C3, High-magnification image of a neurobiotin-filled SPA interneuron (left, arrow) and the corresponding GFP confocal image (right) suggest that the targeted SPA interneuron is coupled with another interneuron by a gap junction. D1, Patch-clamp recording in current-clamp mode at resting membrane potential $(0 \mathrm{pA}$, top red trace) of the targeted SPA interneuron in $\boldsymbol{C}$, the corresponding calcium fluorescence trace (middle), and histogram (bottom) indicating the fraction of active cells as a function of time: each blue peak represents a GDP. Note that the occurrence of a GDP switches off the calcium plateau, as previously shown (Crépel et al., 2007). D2, Recurrent membrane potential oscillations characteristic of SPA cells from the same SPA interneuron that further supports gapjunction coupling by the presence of spikelets; shown on an expanded time scale below.

Here we study the morphophysiological changes in GABAergic interneurons at the transition period between SPA- and GDPdominated networks in the CA3 region of the mouse hippocampus. We use a compound approach that combines chronic daily multineuron calcium imaging, targeted currentand voltage-clamp recordings, inducible genetic fate mapping, and morphological analysis. We show that various types of GABAergic neurons are involved in SPAs and that the transition to a GDP activity pattern occurs during the first postnatal week. We demonstrate that the time when this transition occurs is correlated to the temporal embryonic origin of individual interneurons. Moreover, SPA-interneurons switch their morpho- physiological properties coincident with them receiving GDPs. The physiological diversity that characterizes the GABAergic neuron population only emerges once the SPA-to-GDP transition is completed. Together, these results show that the SPA-toGDP network sequence is reflected at the single-cell level in the morphophysiological evolution of GABAergic neurons.

\section{Materials and Methods}

Acute slice preparation and calcium imaging. All animal use protocols were performed under the guidelines of the French National Ethic Committee for Sciences and Health report on "Ethic Principles for Animal Experimentations" in agreement with the European Community Directive 86/609/EEC. Horizontal hippocampal slices (380 $\mu \mathrm{m}$ thick) were prepared from 1- to 7-d-old (P1-P7) GAD67-green fluorescent protein-knock-in (GAD67-GFP-KI) mice (Tamamaki et al., 2003) or tamoxifen-treated

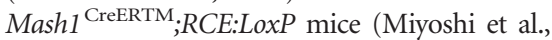
2010) using a vibratome (microtomes VT1200 S; Leica Microsystems) in ice-cold oxygenated modified artificial CSF $\left(0.5 \mathrm{~mm} \mathrm{CaCl}_{2}\right.$ and $7 \mathrm{~mm}$ $\mathrm{MgSO}_{4} ; \mathrm{NaCl}$ replaced by an equimolar concentration of choline). Slices were then transferred and allowed to recover $(\sim 1 \mathrm{~h})$ in oxygenated normal ACSF containing the following (in mM): 126 $\mathrm{NaCl}, 3.5 \mathrm{KCl}, 1.2 \mathrm{NaH}_{2} \mathrm{PO}_{4}, 26 \mathrm{NaHCO}_{3}, 1.3$ $\mathrm{MgCl}_{2}, 2.0 \mathrm{CaCl}_{2}$, and 10 D-glucose, $\mathrm{pH}$ 7.4. For AM-loading, slices were incubated in $2.5 \mathrm{ml}$ of oxygenated ACSF with $25 \mu$ l of a 1 mM fura- 2 AM solution (Invitrogen; in 100\% DMSO) for 20-30 min. Slices were incubated in the dark, and the incubation solution was maintained at $35-37^{\circ} \mathrm{C}$. During imaging, slices were perfused at a rate of 4 $\mathrm{ml} / \mathrm{min}$ with continuously aerated $\left(95 \% \mathrm{O}_{2} / 5 \%\right.$ $\mathrm{CO}_{2}$ ) normal ACSF at $35-37^{\circ} \mathrm{C}$. Imaging was performed with a multibeam two-photon laser scanning system (Trimscope; LaVision Biotec) coupled to an Olympus microscope as previously described (Crépel et al., 2007). Images were acquired through a CCD camera (La Vision Imager $3 \mathrm{QE})$, which typically resulted in temporal resolution of $\sim 100 \mathrm{~ms}(2 \times 2$ binning, pixel size: 600 $\mathrm{nm})$. Slices were imaged using a lowmagnification, high-numerical-aperture objective (20×, NA 0.95; Olympus). The size of the imaged field was typically $430 \times 380 \mu \mathrm{m}^{2}$. Imaging depth was focused, on average, $80 \mu \mathrm{m}$ below the surface (range: $50-100 \mu \mathrm{m}$ ).

Organotypic slice culture preparation and calcium imaging. Organotypic slices were, with minor changes, prepared from the hippocampus of P3 GAD67-GFP-KI mice according to a previously described procedure (Stoppini et al., 1991). Mice were decapitated after anesthesia and put on ice. Following the removal of the brain, hippocampal sections (400 $\mu \mathrm{m}$ thick) were prepared using a McIlwain tissue chopper (Stoerling) and collected in a PBS solution supplemented with $0.5 \%$ D-glucose. Slices were then placed onto Millicell-CM culture inserts $(0.4 \mu \mathrm{m}$; Millipore). The inserts were placed into $35 \mathrm{~mm}$ culture dishes with $1 \mathrm{ml}$ of medium: MEM (Sigma) supplemented with $750 \mu$ l of HEPES $1 \mathrm{M}$, insulin $0.1 \mathrm{mg} / \mathrm{L}$ (solution $5 \mathrm{mgr} / \mathrm{ml} 50 \times$ ), and horse serum (20\%; Invitrogen) at $\mathrm{pH} 7.25-7.29$. Slices were maintained in a cell culture incubator at $37^{\circ} \mathrm{C}$, in an atmosphere containing $5 \% \mathrm{CO}_{2}$. Imaging was performed on the second and third days following slice preparation. Slices were left in their 
initial culture plates with the MEM for the entire experimental protocol. For AM-loading, 1 $\mathrm{ml}$ of regular, but filtered, slice-ACSF as well as $2 \mu \mathrm{l}$ of a 1 mM Fura- 2 AM solution (Invitrogen; in $100 \%$ DMSO) were added for $20-30 \mathrm{~min}$ in the cell culture incubator. Just before calcium imaging, $1 \mathrm{ml}$ of freshly filtered ACSF was added onto the slices and removed just after. For pharmacological experiments, $1 \mathrm{ml}$ of filtered ACSF containing $10 \mu \mathrm{M}$ bumetanide (Sigma) was added onto the slice. The culture plate was kept in the cell culture incubator for $15 \mathrm{~min}$ before the imaging session. Each culture plate that contained slices to be chronically imaged was manipulated under a sterile atmosphere to avoid culture contamination. The microscope objective was sterilized before each imaging session.

Analysis. As previously described (Crépel et al., 2007), analysis of the calcium activity was performed with custom-made software written in Matlab (MathWorks). This program aimed at the automatic identification of loaded cells and at measuring their fluorescence as a function of time. The calcium signal from each cell was determined as the average fluorescence within the contour of that cell, measured as a function of time. MiniAnalysis software (Synaptosoft) signal-processing algorithms were used to detect the onsets and offsets (time of half-amplitude decay) of calcium signals within the traces of individual cells. Active cells were considered to be neurons exhibiting at least one calcium event within the period of recording. The frequency of a network pattern was the averaged time interval between two peaks of synchronous activity. The amplitude of a network pattern in a given movie was the average of the maximum number of cells coactive in each peak of synchrony across the movie. To identify peaks of synchronous activity that included more cells than expected by chance, we used interval reshuffling (randomly reordering of intervals between events for each cell) to create a set of surrogate event sequences. Reshuffling was performed 1000 times for each movie, and a surrogate histogram was constructed for each reshuffling. The threshold corresponding to a significance level of $p<0.05$ was estimated as the number of coactive cells exceeded in a single frame in only $5 \%$ of these histograms. The entire procedure could be performed on-line sufficiently quickly to identify cells for targeted patch-clamp recordings.

For the analysis of organotypic slice experiments, the difficulty was to track the position of single-cells across days as the soma location of neurons tends to move, mostly due to the continuous slice flattening that occurs in the culture plate. Typically, focal planes from three different depths are necessary to recover all the cells imaged within a $1 \mathrm{~d}$ interval. Easily recognizable visual cues provided by the GFP labeling were used to ensure that imaging was always from the same set of neurons.

Electrophysiology. Neurons were patch-clamp recorded in the wholecell configuration. Microelectrode resistance was 4-8 M $\Omega$. Uncompensated access resistance was monitored throughout the recordings. Values $<20 \mathrm{M} \Omega$ were considered acceptable and the results were discarded if it

A

C
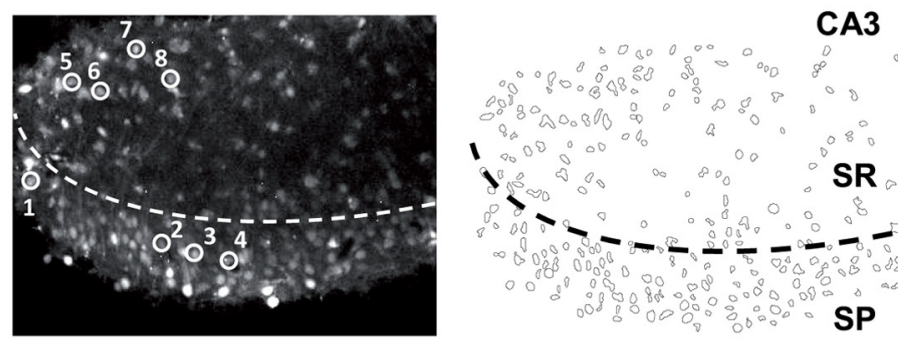

$\mathrm{D}+1$

\section{Glutamatergic cells}

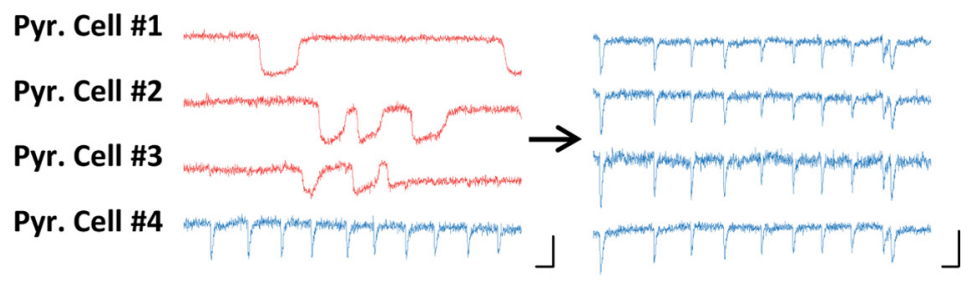

GABAergic cells
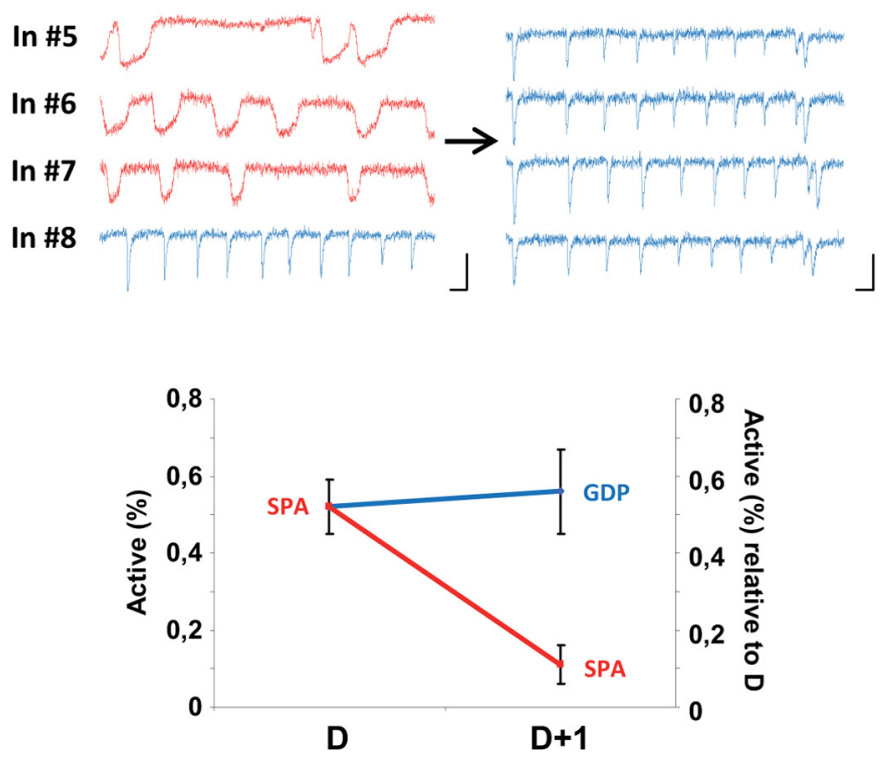

Figure 2. SPA cells develop into GDP cells as the hippocampal network matures. $\boldsymbol{A}$, Two-photon calcium fluorescence image (left) and contour map of the cells imaged (right) in the CA3 region of an organotypic hippocampal slice prepared from a $\mathrm{P} 4$ mouse. SR, Stratum radiatum; SP, Stratum pyramidale. B, Calcium fluorescence traces as a function of time of four pyramidal cells (Pyr. Cell $\# 1-4$ ) and four interneurons (In \#5-8), circled in $A$ and monitored after $2 \mathrm{~d}$ in culture (Day $D)$ and $1 \mathrm{~d}$ later $(D+1)$. Scale bars: $x$-axis, 10 s; $y$-axis, 20\% DF/F. Note that pyramidal cells $1-3$ and interneurons $5-7$ first produce calcium plateaus characteristic of SPAs at $D$ and display synchronous fast calcium transients associated with GDPs at $D+1$. C, Graph showing the fraction of SPA cells relative to all active cells ( $y$-axis on the left) at $D$ and the fraction of SPA and GDP cells relative to the number of SPA cells at $D$ on $D+1$ ( $y$-axis on the right; $n=4$ slices, 520 neurons). Note that the proportion of SPA cells at $D$ that become involved in GDPs the next day is greater than that still producing SPAs at $D+1$ ( $56 \pm 5 \%$ vs $11 \pm 10 \%)$.

changed by $>20 \%$. Whole-cell measurements were filtered at $3 \mathrm{kHz}$ using a patch-clamp amplifier (EPC10; HEKA). Recordings were digitized on-line $(20 \mathrm{kHz})$ with a Labmaster interface card to a personal computer and acquired using Axoscope 7.0 software (Molecular Devices). Synchronization between optical and electrical signals was achieved by feeding simultaneously the Labmaster interface card with the trigger signals for each movie frame and the electrophysiological recordings. For voltage-clamp experiments, the composition of the intracellular solution was as follows: $120 \mathrm{~mm}$ Cs-gluconate, $10 \mathrm{~mm} \mathrm{MgCl}_{2}, 0.1 \mathrm{~mm}$ 
A

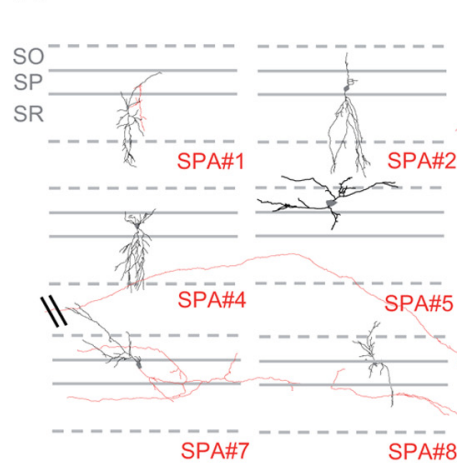

B

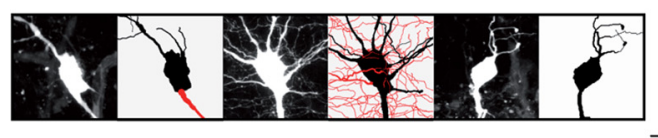

C
SPA

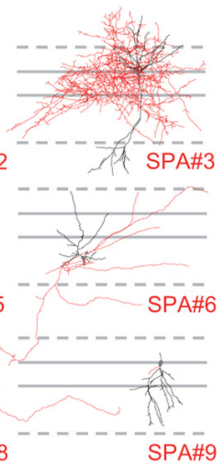

GDP

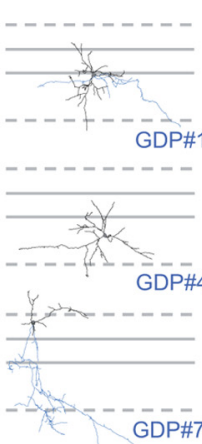

GDP\#7
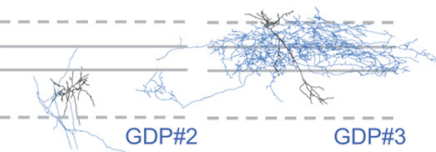

GDP\#3

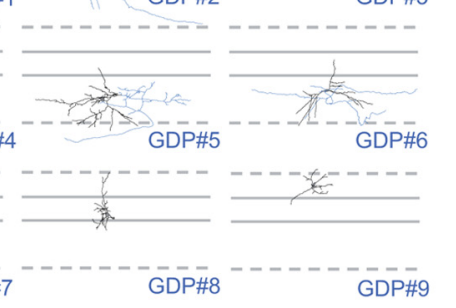

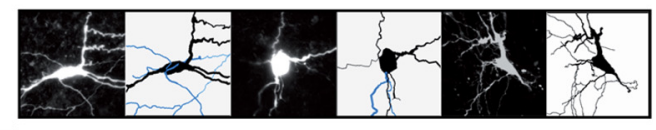
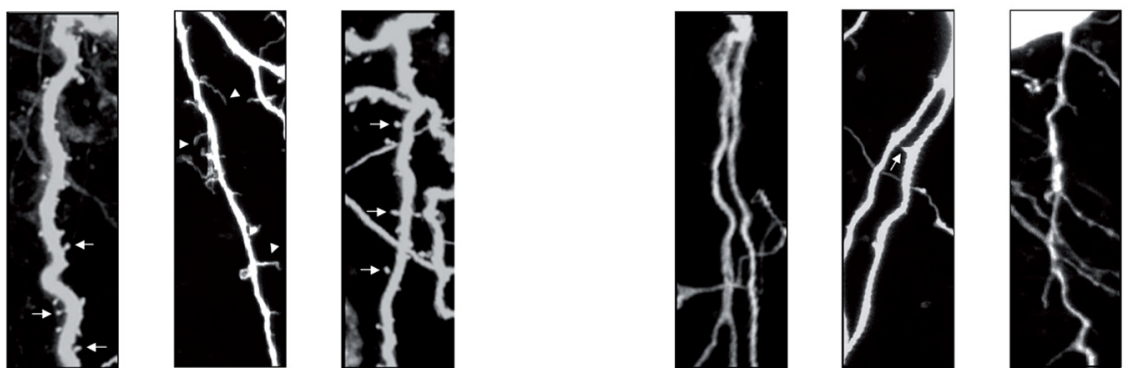

Figure 3. SPA and GDP interneurons are morphologically diverse, with SPA interneurons displaying larger somata and a higher density of membrane protrusions. Neurolucida reconstructions of 18 Neurobiotin-filled neurons, nine SPA interneurons (left) and nine GDP interneurons (right) from P4 GAD67-KI-GFP mice. Both groups are morphologically diverse (black, dendrites and soma; red, axons for SPA interneurons; blue, axons for GDP interneurons). SR, Stratum radiatum; SP, Stratum pyramidale; SO, Stratum oriens. Scale bar, $50 \mu \mathrm{m}$. B, Confocal fluorescence images and the corresponding neurolucida reconstructions of the three SPA (left) and three GDP interneurons (right) illustrated in $\boldsymbol{A}$ at a high magnification centered on the soma. Note that the soma of SPA interneurons is larger than that of GDP interneurons. Scale bar, $50 \mu \mathrm{m}$. C, Confocal fluorescence images of a fragment of second-order dendritic tree for three SPA (left) and three GDP interneurons (right) illustrated in $\boldsymbol{A}$. Scale bar, $5 \mu \mathrm{m}$. Note that the dendrites of SPA interneurons are covered with numerous protrusions of various shapes, i.e., round and small (arrows) or elongated (arrowheads).

$\mathrm{CaCl}_{2}, 1$ mM EGTA, $5 \mathrm{~mm} \mathrm{Na}$ adenosine triphosphate, 10 mM HEPES. With this solution, glutamate receptor-mediated postsynaptic currents (PSCs) reversed at $+10 \mathrm{mV}$ while $\mathrm{GABA}_{\mathrm{A}} \mathrm{R}$-mediated PSCs reversed at $-60 \mathrm{mV}$. Miniature IPSC and EPSCs (mIPSCs and mEPSCs) were collected in the presence of TTX ( $1 \mu \mathrm{M}$; Tocris Bioscience) at a holding potential of $+10 \mathrm{mV}$ and of $-60 \mathrm{mV}$, respectively. Recordings were also performed at $+40 \mathrm{mV}$ to isolate NMDAR-mediated EPSCs. For currentclamp recordings, the intracellular solution contained the following: 130 mm K-methyl-SO ${ }_{4}, 5 \mathrm{~mm} \mathrm{KCl}, 5 \mathrm{~mm} \mathrm{NaCl}, 10 \mathrm{~mm}$ HEPES, $2.5 \mathrm{~mm} \mathrm{Mg-}$ ATP, and 0.3 mM GTP. No correction for liquid junction potential was applied. The osmolarity was $265-275 \mathrm{mOsm}, \mathrm{pH}$ 7.3. Recordings were analyzed using the MiniAnalysis software (Synaptosoft).

Morphological analysis. Slices were processed for the detection of Neurobiotin-filled neurons as follows: briefly, slices were fixed overnight at $4^{\circ} \mathrm{C}$ in Antigenfix (Diapath). After several rinses in PBS containing $0.3 \%$ Triton X-100 (PBST), slices were incubated overnight at room temperature in $1 / 1000$ streptavidin coupled to cy 3 diluted in PBST. Slices were rinsed in PBS before mounting between slide and coverslide in Fluoromount (Southern Biotech). Post hoc analysis was performed using a confocal microscope. Stacks of optical sections were collected for computer-assisted neuron reconstructions.

Eighteen neurons were reconstructed with a computer-assisted system attached to a microscope (Neurolucida software; MicroBrightfield). Morphological variables included the following: total dendritic and axonal lengths, total dendritic and axonal surfaces, and total number of dendritic and axonal terminals. The total surface of dendrites or axons (branched structures) is an estimated value calculated by the software. The total length of dendrites or axons is the sum of the lengths of all the branches. We also performed a Sholl analysis to determine the distribution of the number of dendritic intersections with circles of increasing radius ( $20 \mu \mathrm{m}$ steps) centered at the cell's soma.

For each neuron, the linear density of dendritic protrusions was calculated in a $20 \mu \mathrm{m}$ segment length from second-order dendritic branches.

Mice for inducible genetic fate mapping. Double-homozygous Mash1BAC CreER/CreER/RCE:LoxP $P^{+/+}$(Miyoshi et al., 2010) male mice were crossed with 7- to 8-week-old wild-type Swiss females (a gift from Comité d'Entreprise (Works Council) Janvier, Le Genest-Saint-Isle, France) for offspring production. To induce CreER activity, we administered a tamoxifen solution (Sigma) by gavaging (force-feeding) pregnant mice with a silicon-protected needle (Fine Science Tools). We used $2 \mathrm{mg}$ of tamoxifen solution per $30 \mathrm{~g}$ of body weight, prepared at $10 \mathrm{mg} / \mathrm{ml}$ in corn oil (Sigma). Pregnant females crossed with Mash1BAC ${ }^{\text {CreER/ }}$ CreER/RCE:LoxP $P^{+/+}$males were gavaged at E11.5 and E18.5 to label earlyand late-expressing Mash1 precursors, respectively, in the embryos named Mash1 ${ }^{\text {CreER }}$;RCE:LoxP mice (Picardo et al., 2011).

Statistical analysis. Experimental values are given as mean \pm SEM. Statistical significance between means was calculated using Student's $t$ test or Mann-Whitney rank sum test in the case where normality test failed. $p<0.05$ was considered significant.

\section{Results}

GABAergic interneurons are involved in SPAs

We hypothesized that SPAs could be a central step for the maturation of interneurons, given that GABAergic transmission is essential for the generation of GDPs (Ben-Ari et al., 1989; Gara- 
schuk et al., 1998; Sipilä et al., 2005; Bonifazi et al., 2009), and that SPAs occur just before their emergence (Crépel et al., 2007; Allene et al., 2008). However, it remains unknown whether SPAs similarly involve glutamatergic and GABAergic neurons. To address this issue, we quantified the proportion of GABAergic cells producing calcium plateaus. We focused on P1-P5 and imaged the CA3 hippocampal region of GAD67-GFP-KI mouse slices in which GABAergic cells are labeled with GFP (Tamamaki et al., 2003). The contour maps corresponding to GFP-positive neurons were superimposed on the distribution of neurons producing SPAs (see Materials and Methods, above; Fig. 1). We found that cells involved in coordinated activity patterns near birth (P1) comprise mainly GABAergic neurons, with $\sim 25 \%$ of interneurons involved either in SPAs or GDPs $(16 \pm 4 \%$ and $9 \pm 9 \%$, respectively; $n=6$ slices; Fig. 1 B2) compared with $<5 \%$ of pyramidal cells involved in any type of activity (Fig. 1B2). Later, in both neuronal populations, the fraction of cells involved in coordinated activities increases across the first postnatal week (Fig. 1B2). Specifically focusing our analysis on SPAs, we found that half of the SPA neurons were GABAergic at P1 (51 $\pm 9 \%$ of SPA cells were GFP + at P1, $n=6$ slices; Fig. $1 B 1$ ) and that the participation of interneurons to SPAs declined abruptly within a few days ( $20 \pm 12 \%$ of SPA cells were GFP + at P3, $n=5$ slices; Fig. 1B1). Ultimately, at P7, almost all GABAergic neurons participated in GDPs ( $87 \pm 7 \%$ of GFP + cells were GDP neurons at P7, $n=6$ slices). Targeted patch-clamp recordings confirmed that GFP-positive SPA neurons indeed produced the characteristic membrane potential oscillations associated with SPAs (Fig. 1D). Moreover, in a few cases, we observed dye labeling of two interneurons as well as spikelets, indicating a putative gap-junction coupling between SPA cells (Fig. 1C,D2). In contrast, in more mature slices (P5), almost $90 \%$ of SPA cells were glutamatergic neurons (on average: $11 \pm 3 \%$ SPA cells were GFP,$+ n=9$ slices; Fig. 1B1). We conclude that GABAergic neurons participate in the production of SPAs at different developmental stages, with their involvement decreasing over time until the end of the first postnatal week, when almost all GABAergic neurons are involved in GDPs.

\section{SPA cells develop into GDP cells as the hippocampal network matures}

Given that developmental cell loss has been reported at early postnatal stages (Buss and Oppenheim, 2004), SPAs could be selectively expressed by transient neuronal populations or, alternatively, be a transient activity step occurring during the maturation of most neurons. To directly address this question, we have performed daily multineuron calcium imaging following the same neuronal populations in the CA3 region of organotypic hippocampal slices prepared from GAD67-GFP-KI mice. To this end, we have designed an experimental procedure to avoid culture contamination through repetitive imaging sessions using multibeam two-photon calcium microscopy (see Materials and Methods, above). Organotypic hippocampal slice cultures provide a robust model to chronically study the maturation of coherent activity patterns since they produce similar network dynamics, as previously observed in acute slices (Crépel et al., 2007). Indeed, spontaneous network dynamics similar to GDPs in terms of both their calcium dynamics and pharmacological characteristics (Crépel et al., 2007) could be recorded in every organotypic slice ( $n=12$ slices): they consisted of fast calcium events occurring simultaneously in many neurons within one movie frame (Crépel et al., 2007) at $0.04 \pm 0.01 \mathrm{~Hz}(n=12$ slices; Fig. 2). These dynamics were dependent on the excitatory actions
Table 1. Comparison of the morphophysiological properties of SPA and GDP interneurons

\begin{tabular}{|c|c|c|}
\hline & SPA & GDP \\
\hline \multicolumn{3}{|l|}{ Electrophysiological properties } \\
\hline$R_{\mathrm{m}}(\mathrm{M} 0 \mathrm{hm})$ & $511 \pm 61(n=11)^{*}$ & $779 \pm 72(n=6)$ \\
\hline$C_{m}(p F)$ & $78 \pm 8(n=11)^{*}$ & $48 \pm 5(n=6)$ \\
\hline$V_{\text {rest }}(\mathrm{mV})$ & $-48.4 \pm 5(n=6)^{*}$ & $-74.5 \pm 3(n=6)$ \\
\hline AP amplitude (mV) & $52 \pm 5(n=11)^{*}$ & $95 \pm 6(n=6)$ \\
\hline AP threshold (mV) & $-54 \pm 3(n=11)$ & $-62 \pm 4(n=6)$ \\
\hline AP duration (ms) & $4 \pm 0.4(n=11)^{*}$ & $2 \pm 0.1(n=6)$ \\
\hline \multicolumn{3}{|l|}{ Postsynaptic currents } \\
\hline \multicolumn{3}{|l|}{ Spontaneous IPSCS } \\
\hline Amplitude (pA) & $35 \pm 4(n=5)^{*}$ & $29 \pm 9(n=5)$ \\
\hline Frequency (Hz) & $1.9 \pm 0.4(n=5)$ & $2.6 \pm 0.8(n=5)$ \\
\hline Rise (ms) & $1.4 \pm 0.2(n=5)^{*}$ & $3.2 \pm 1.2(n=5)$ \\
\hline Decay (ms) & $10 \pm 2(n=5)^{*}$ & $24 \pm 5(n=5)$ \\
\hline \multicolumn{3}{|l|}{ mIPSCs } \\
\hline Amplitude (pA) & $33 \pm 4(n=5)^{*}$ & $21 \pm 6(n=5)$ \\
\hline Frequency (Hz) & $0.6 \pm 0.1(n=6)$ & $0.7 \pm 0.5(n=6)$ \\
\hline Rise (ms) & $1.4 \pm 0.2(n=6)$ & $2.5 \pm 1(n=6)$ \\
\hline Decay (ms) & $10 \pm 1.5(n=6)$ & $16 \pm 4(n=6)$ \\
\hline \multicolumn{3}{|l|}{ Spontaneous EPSCS } \\
\hline Amplitude (pA) & $23 \pm 5(n=5)$ & $29 \pm 7(n=5)$ \\
\hline Frequency (Hz) & $1.6 \pm 0.4(n=5)$ & $4 \pm 2(n=5)$ \\
\hline Rise (ms) & $0.8 \pm 0.2(n=5)$ & $1.6 \pm 0.3(n=5)$ \\
\hline Decay (ms) & $3.5 \pm 1(n=5)$ & $9.2 \pm 3(n=5)$ \\
\hline \multicolumn{3}{|l|}{ mEPSCs } \\
\hline Amplitude (pA) & $30 \pm 6(n=6)^{*}$ & $16 \pm 1.2(n=5)$ \\
\hline Frequency (Hz) & $0.5 \pm 0.1(n=6)$ & $0.4 \pm 0.14(n=5)$ \\
\hline Rise (ms) & $0.6 \pm 0.1(n=6)$ & $1.8 \pm 0.5(n=5)$ \\
\hline Decay (ms) & $3 \pm 0.7(n=6)^{*}$ & $9.4 \pm 1.4(n=5)$ \\
\hline \multicolumn{3}{|l|}{ Morphology } \\
\hline Cell body area $\left(\mu \mathrm{m}^{2}\right)$ & $165 \pm 21(n=9)^{*}$ & $105 \pm 10(n=9)$ \\
\hline \multicolumn{3}{|l|}{ Dendrites } \\
\hline Length $(\mu \mathrm{m})$ & $1422 \pm 199(n=9)$ & $1206 \pm 154(n=9)$ \\
\hline Surface $\left(\mu \mathrm{m}^{2}\right)$ & $41667 \pm 7163(n=9)$ & $34602 \pm 7783(n=9)$ \\
\hline Nodes & $21.4 \pm 3(n=9)$ & $22.9 \pm 4(n=9)$ \\
\hline Protrusions (number/ $\mu \mathrm{m}$ ) & $0.25 \pm 0.05(n=22)^{*}$ & $0.14 \pm 0.05(n=16)$ \\
\hline \multicolumn{3}{|l|}{ Axon } \\
\hline Length & $6909 \pm 4863(n=5)$ & $5242 \pm 3464(n=6)$ \\
\hline Surface & $403776 \pm 226349(n=5)$ & $182316 \pm 72865(n=6)$ \\
\hline Nodes & $148 \pm 135(n=5)$ & $145 \pm 115(n=6)$ \\
\hline
\end{tabular}

of GABA, as they were blocked by the selective NKCC1 antagonist bumetanide (in bumetanide $(10 \mu \mathrm{M})$, the frequency and amplitude of GDPs decreased to $11 \pm 11 \%$ and $20 \pm 20 \%$ of control, respectively; $n=5$ slices, $p<0.05$; data not shown). Calcium plateaus corresponding to SPAs could also be recorded and involved $27 \pm 6 \%$ of imaged neurons on average ( $n=12$ slices). We next focused on the fate of individual SPA cells and surprisingly found that, after $1 \mathrm{~d}$, many SPA cells stopped producing calcium plateaus and participated in GDPs. Indeed, after $1 \mathrm{~d}$ in culture, the fraction of SPA cells involved in GDPs significantly increased, whereas the fraction of SPA cells still producing plateaus decreased (to $56 \pm 5 \%$ and $11 \pm 1 \%$, respectively; $n=4$ slices, $n=$ 520 neurons; Fig. 2). If we focus our analysis on the GABAergic neuron population, we find that $40 \%$ of GFP-positive SPA cells (i.e., SPA interneurons) transit to the GDP stage within $1 \mathrm{~d}$ (39 \pm $13 \%, n=4$ slices, $n=96$ neurons; Fig. 2 ), whereas the fraction of SPA interneurons still producing plateaus decreased by $>60 \%$ (to $39 \pm 8 \%$ ). In addition, almost all interneurons starting to participate in GDPs on the second imaging session had been involved in SPAs on the previous day ( $86 \%$ of newly detected GFP + GDP cells originate from GFP + SPA cells, $n=44$ GFP + 
A

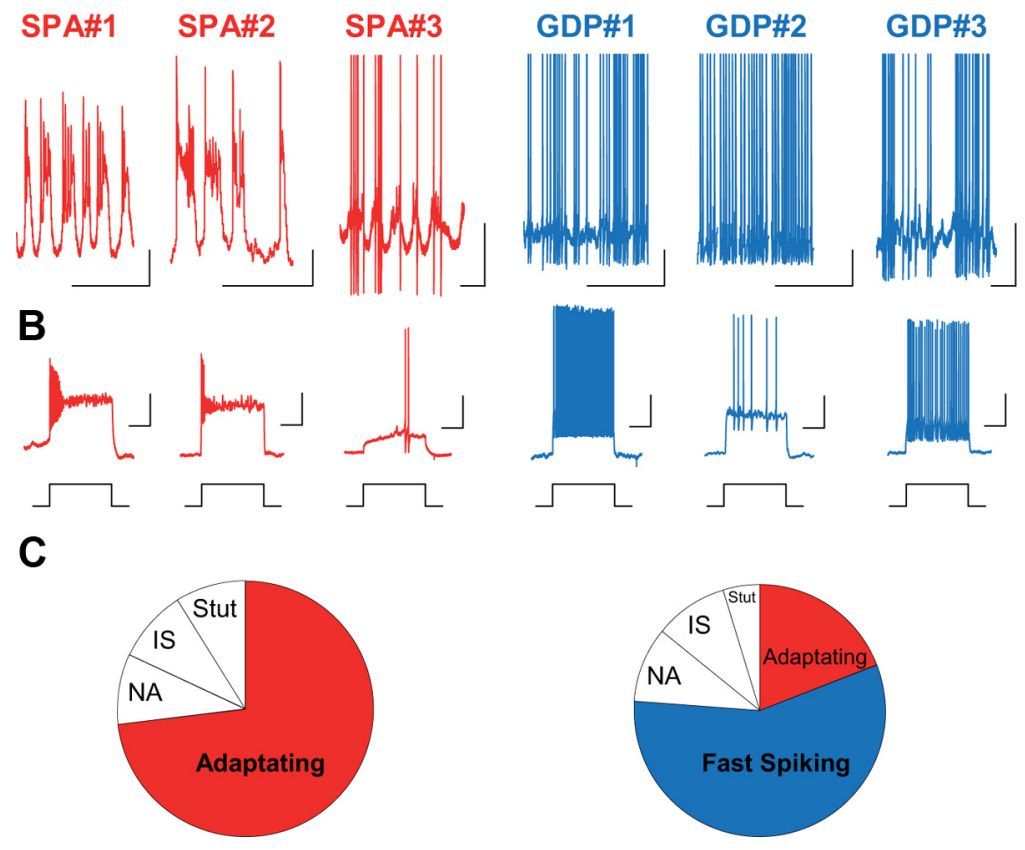

Figure 4. Different SPA interneurons display similar intrinsic electrophysiological properties; firing diversity only emerges in GDP interneurons. $A$, Electrophysiological recordings in current-clamp mode at resting membrane potential $(I=0 \mathrm{pA})$ in the presence of blockers of synaptic transmission (10 $\mu \mathrm{m}$ bicuculline, $10 \mu \mathrm{M}$ NBQX, $40 \mu \mathrm{m}$ APV) from the three SPA interneurons (SPA\#1-3) and the three GDP interneurons (GDP\#1-3) illustrated in Figure 3A from P4 GAD67-KI-GFP mice. Scale bars: $x$-axis, 1 s; $y$-axis, $20 \mathrm{mV}$. B, Firing patterns in response to a depolarizing current step (+40 pA). Scale bars: $x$-axis, $5 \mathrm{~s} ; y$-axis, $10 \mathrm{mV}$. Note that at the same developmental stage (P4), a basket-like SPA interneuron (SPA\#3; Fig. 3A) presents a strongly delayed and adapting firing pattern while a basket-like GDP interneuron (GDP\#3; Fig. 3A) displays a fast-spiking pattern. C, Diagram representing the distribution of different firing patterns recorded in SPA interneurons $(n=11)$. NA, Non-adaptating; IS, irregular-spiking; Stut, stuttering. Note that SPA interneurons display similar intrinsic electrophysiological properties with an adapting firing pattern and recurrent membrane potential oscillations at resting membrane potential; GDP interneurons display a diversity of firing patterns.

cells). We therefore conclude that a portion of GDP interneurons originate from SPA interneurons and that the fate of many SPA interneurons is to start participating in GDPs as development proceeds.

\section{GABAergic SPA and GDP cells possess variable morphologies, with SPA interneurons displaying a larger soma size and dense membrane protrusions}

We next wondered whether the transition between SPA and GDP activity patterns may be paralleled by changes in the morphophysiological properties of GABAergic interneurons. To analyze morphological features, GABAergic SPA and GDP cells were targeted for neurobiotin-filling based on their calcium kinetics, first at $\mathrm{P} 4$, a stage when both patterns are expressed in comparable proportions within the CA3 hippocampal network. GFP-positive cells were specifically targeted and immunohistochemically processed post hoc. Of 53 morphologically recovered interneurons, nine SPA and nine GDP interneurons were reconstructed for morphometric analysis (Fig. 3). Although at P4, SPA and GDP interneuron populations were quite morphologically immature, they still displayed considerable diversity with various cell body locations and different dendritic and axonal arborization patterns (Fig. 3). The soma of SPA and GDP interneurons was located in stratum oriens (in 22\% and 33\% of the cases, respectively; $n=53)$, radiatum $(22 \%$ and $55 \%$, respectively; $n=53$ ), or pyramidale ( $55 \%$ and $11 \%$, respectively; $n=53$ ). Approximately half of the interneurons in both groups displayed a very small axon, if any. Among the interneurons with a more developed axonal arborization, we observed two SPA interneurons with puta- tive perisomatic, basket-like axonal morphology (one is reconstructed in Fig. 3) and two putative long-range SPA interneurons (Fig. 3). Concerning GDP interneurons, we found four cells with putative perisomatic, basket-like axonal morphology (one is reconstructed in Fig. 3) and five cells with more local and less arborized axons (Fig. 3 ). The presence of basket-like interneurons in both groups suggests that this interneuron subtype likely transits from a SPA to a GDP state. We also quantified dendritic and axonal lengths, nodes, and surfaces (Table 1) and performed a Sholl analysis (data not shown). In comparing SPA and GDP interneurons, none of the parameters measured were significantly different. Even within the same putative morphological subgroup of basket-like interneurons, there was no striking significant difference between cell morphologies (Fig. 3). However, SPA interneurons could be distinguished from GDP interneurons by two features. First, on average, they exhibited a significantly larger soma size $\left(165 \pm 21 \mu \mathrm{m}^{2}, n=\right.$ 9 SPA cells vs $105 \pm 10 \mu \mathrm{m}^{2}, n=9$ GDP cells, $p<0.05$; Table 1, Fig. $3 B$ ). Second, their dendrites (and sometimes their soma; Fig. $3 C$ ) tended to be covered with numerous protrusions. The linear density of these processes was quantified (see Materials and Methods, above) and, on average, significantly higher in SPA than GDP interneurons $(0.25 \pm 0.05$ protrusions $/ \mu \mathrm{m}, n=22$ cells and $0.14 \pm 0.05$ protrusions $/ \mu \mathrm{m}, n=$ 16 cells, respectively; $p<0.05$; Fig. $3 C$ ). This is surprising given that, in contrast to glutamatergic cells and with a few exceptions, the dendritic arbor of most GABAergic interneurons is aspiny.

We conclude that both SPA and GDP interneuron populations likely comprise a variety of morphological cell types that are largely at similar stages of dendritic and axonal development. However, SPA interneurons display certain characteristics, including a larger cell body and membrane protrusions.

\section{While different subtypes of GABAergic SPA cells display similar intrinsic electrophysiological properties, firing diversity only emerges in GABAergic GDP cells}

We next targeted SPA and GDP interneurons at P4 for currentand voltage-clamp recordings and characterized their intrinsic and synaptic electrophysiological properties. As expected (Crépel et al., 2007), in current-clamp mode, at resting membrane potential $\left(V_{\text {rest }}\right)$, SPA interneurons displayed recurrent membrane potential oscillations, which were not affected by synaptic transmission blockade using $\mathrm{GABA}_{\mathrm{A}} \mathrm{R}, \mathrm{AMPAR}$, or NMDAR antagonists ( $10 \mu \mathrm{m}$ bicuculline, $10 \mu \mathrm{M}$ NBQX, $40 \mu \mathrm{M}$ D-APV, $n=14$ cells; Fig. 4). Most GDP interneurons were also spontaneously firing at $V_{\text {rest }}$ in the presence of synaptic blockers; however, in contrast to SPA interneurons, they tonically fired action potentials (Fig. 4). We also investigated the firing patterns of interneurons in response to depolarizing and hyperpolarizing current steps. We found that almost all SPA interneurons displayed a strongly adapting firing pattern $(72 \%, n=11$ SPA interneurons; Fig. 4), whereas GDP interneurons presented a variety of firing 
patterns (Fig. 4) that could be classified, according to the Petilla nomenclature (Ascoli et al., 2008), as fast-spiking $(n=12)$, nonadapting $(n=2)$, adapting $(n=4)$, irregular-spiking $(n=2)$, or stuttering $(n=1)$. Basket-like GDP interneurons presented a fast-spiking pattern, whereas spiking in basket-like SPA cells was strongly delayed and adapting (Fig. 4). In addition, $V_{\text {rest }}$ and action potential (AP) amplitude and duration were significantly different between SPA and GDP interneurons: SPA cells displayed a more depolarized $V_{\text {rest }}$ and a broader and smaller AP (Table 1), all features indicating an immature stage of development (Moody and Bosma, 2005; Doischer et al., 2008; Okaty et al., 2009). Moreover and in agreement with the morphological results concerning the cell body size, the membrane resistances $\left(R_{\mathrm{m}}\right)$ and capacitances $\left(C_{\mathrm{m}}\right)$ of SPA and GDP interneurons were significantly different. SPA interneurons displayed smaller membrane resistances and higher membrane capacitances when compared with GDP interneurons (Table 1). In contrast, the fraction of SPA and GDP interneurons displaying an $h$ current evoked by hyperpolarizing current steps was not different $(83 \%, n=7$ neurons and $90 \%, n=20$ neurons, respectively).

\section{Synaptic inputs differ between SPA and GDP interneurons}

Since GDPs are synapse-driven network events, whereas SPAs do not depend on synaptic transmission for their generation, we hypothesized that GDP interneurons receive more synaptic inputs than SPA interneurons. As a first step toward addressing this issue, at P4, we recorded spontaneous and miniature EPSCs and IPSCs in SPA or GDP interneurons. Surprisingly, on average, the frequency and kinetics of spontaneous and miniature EPSCs and IPSCs impinging onto SPA and GDP interneurons were not significantly different (Table 1). However, the average amplitude of miniature events (mEPSCs and mIPSCs) received by SPA interneurons was significantly larger than that received by GDP interneurons (Table 1, Fig. 5). These large average amplitude values can be attributed to the higher occurrence of large amplitude PSCs in SPA interneurons compared with GDP interneurons, as revealed by PSCs amplitude distribution histograms. Histograms plotting the distribution of $\mathrm{mIPSC} / \mathrm{mEPSC}$ amplitudes of both SPA and GDP interneurons were unimodal but skewed toward larger events in SPA cells (Kolmogorov-Smirnov, $p<0.05)$. Accordingly, mEPSCs and mIPSCs received by SPA interneurons displayed a higher variability as measured by their larger standard deviation ( $\mathrm{SD}=27$ vs 6 , for mEPSCs in SPA vs GDP cells, and SD $=34$ vs 21, for mIPSCs in SPA vs GDP cells; Fig. 5). We conclude that SPA cells receive synaptic inputs of higher amplitude variability with significantly higher average amplitude values than GDP cells.

\section{Cells mature during the SPA stage in accordance with their birth date}

At P4, SPA interneurons share characteristic morphophysiological properties that include membrane protrusions, immature firing patterns, and high amplitude synaptic inputs. We next asked whether these properties could still be found in the few remaining SPA interneurons at later postnatal stages (P7). To address this issue, we targeted five SPA interneurons at P7 and found that their morphophysiological properties were not significantly different from SPA interneurons at P4, with the exception that there was a global increase in the size of neurons (Fig. 6). Hence, if the average cell body area of all neurons, including SPA cells, increased between P4 and P7 (by 120\% as estimated from the area of imaged cell contours), the soma of SPA cells remained significantly larger than that of GDP cells at P7 $\left(207 \pm 4 \mu \mathrm{m}^{2}\right.$,

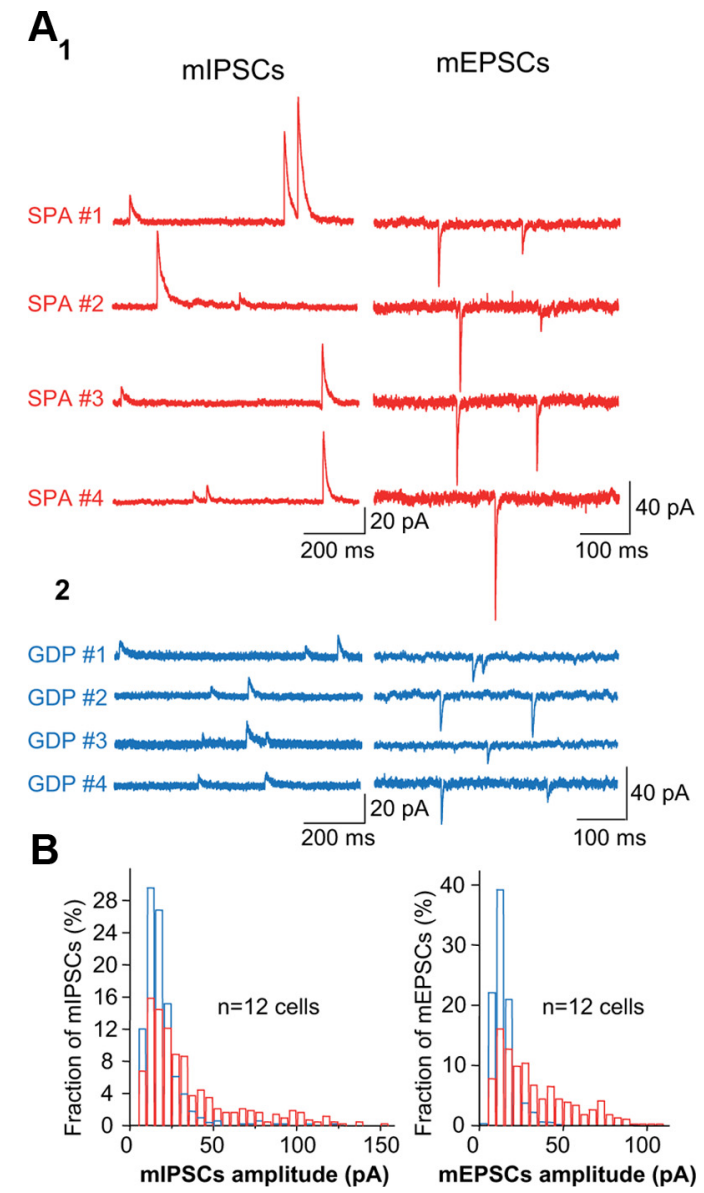

Figure 5. Large amplitude miniature postsynaptic currents are only recorded in SPA cells. A1, GABA ${ }_{A} R$-mediated mIPSCs recorded at $+10 \mathrm{mV}$ and AMPA/KAR-mediated mEPSCs recorded at $-60 \mathrm{mV}$ in the CA3 hippocampal region of P4 GAD67-KI-GFP mice in four SPA interneurons. $\boldsymbol{A 2}$, Same as $\boldsymbol{A} \mathbf{1}$, but in four GDP interneurons. Note the occurrence of high amplitude mIPSC and mEPSCs only in SPA interneurons. $\boldsymbol{B}$, Histograms plotting the pooled distribution of mIPSCs and mEPSCs amplitudes of SPA (red) and GDP (blue) interneurons. Note the strong right skew of both mIPSCs and mEPSCs distributions for SPA interneurons, indicating a large variety of event amplitudes with a significant occurrence of larger events.

$n=3$ and $143 \pm 19 \mu \mathrm{m}^{2}, n=4$, respectively; $\left.p<0.05\right)$. Therefore, we conclude that the morphophysiological features of SPA cells are not likely to be determined by the developmental stage of the network they are embedded into but rather reflect the intrinsic developmental stage of the SPA cell. To further confirm this hypothesis, we analyzed the involvement of interneurons in SPAs according to their birth date. We hypothesized that earlygenerated interneurons (EG-Ins) could have already achieved the SPA-to-GDP transition at P3 when GABAergic cells are still more involved in SPAs than GDPs, whereas late-generated interneurons (LG-Ins) (Picardo et al., 2011) would be more likely to be still involved in SPAs at P7, when the majority of interneurons have transitioned to a GDP stage. We therefore used an inducible genetic fate mapping strategy to label interneurons according to their time of embryonic origin. We combined the $M a s h 1 B A C^{C r e E R}$ driver with the RCE:loxP reporter (Picardo et al., 2011) and through tamoxifen administration at E11.5 and E18.5, two subpopulations of CA3 EG-Ins and LG-Ins, which are specifically derived from the E11.5 and E18.5 caudal ganglionic eminence respectively, are labeled by EGFP (for details, see Materials and Methods, above). At P3, EG-Ins were targeted for electrophysiological recordings. Unfortunately, for unknown reasons, 
A
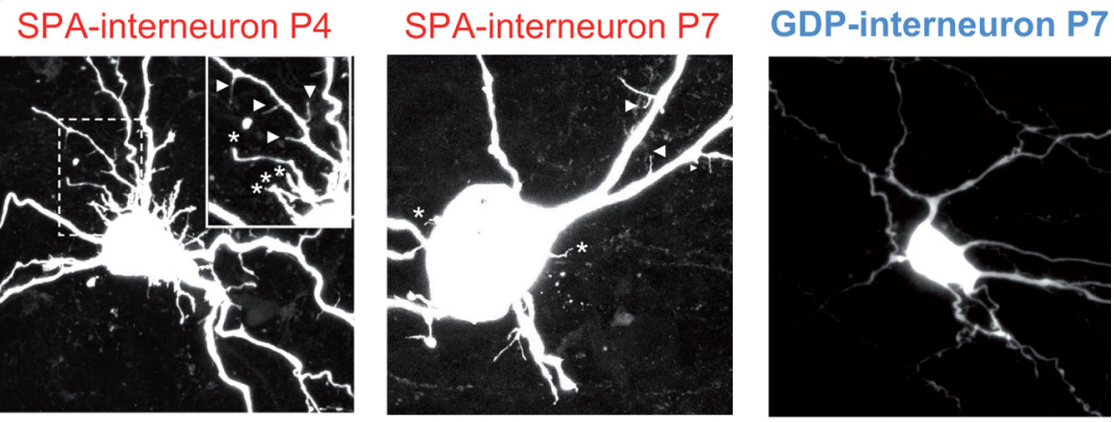

B
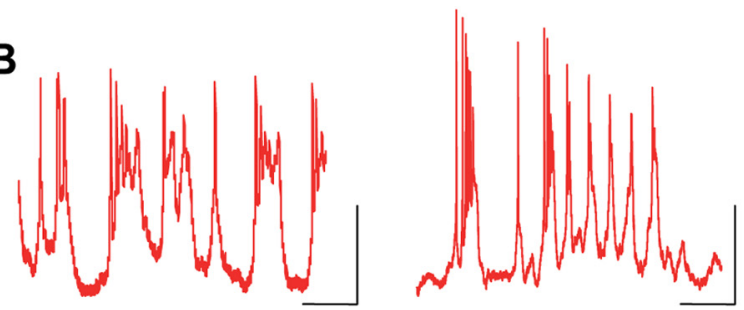

C
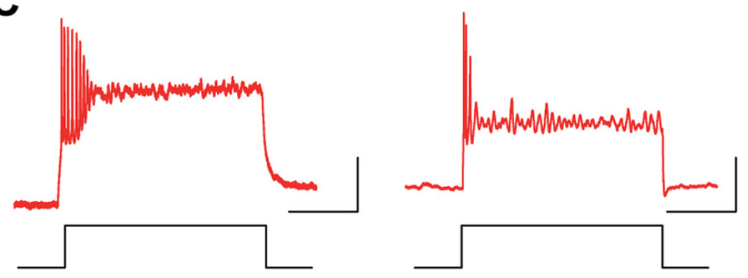

D

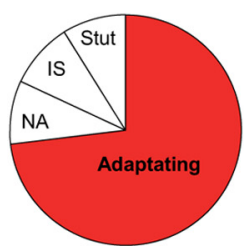

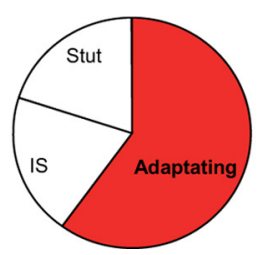

lation, as $43 \%$ of active LG-Ins were detected as SPA cells $(n=7$ cells; Fig. $7 B)$ while SPA cells accounted for $<20 \%$ of all GABAergic neurons $(16 \%, n=91$ neurons, 6 slices; Fig. 7B3). We conclude that EG-Ins already produce GDPs at a period dominated by SPAs, whereas LG-Ins are involved in SPAs over a protracted period of interneuron maturation.

\section{Discussion}

We have examined the correlation between the development of cellular properties in GABAergic interneurons and their participation in spontaneous patterned activities by combining imaging with electrophysiology, morphology, and inducible genetic fate mapping. We find that in parallel to the sequential evolution from SPAs to GDPs, the properties of GABAergic cells mature at anatomical, intrinsic electrophysiological and synaptic levels. This demonstrates that events at the network and cellular level are most likely modulated by reciprocal feedback on one another. However, interneuron involvement in SPAs or GDPs is dependent on their birth: while the morphophysiological characteristics of SPA interneurons are constant at both early and late postnatal stages, late-generated interneurons tend to produce SPAs at later developmental periods and early-generated ones are more likely to receive GDPs during early postnatal periods. These results support a strong correlation between circuit and single-cell maturation.

\section{Within the same network, developing neurons are heterogeneously involved in different activity patterns}

Although network oscillations produced by a specific cellular mechanism dominate at specific stages of development (Allene and Cossart, 2010; Blankenship and Feller, 2010), several types of spontaneous activities coexist within the same network as development progresses. In addition, many cells are silent and thus do not participate in any form of synchronous activity. The present study focuses on a narrow time period of postnatal development that EG-Ins could not be efficiently labeled with the calciumpermeable indicator used here (Fura-2 AM) (Picardo et al., 2011), which precluded determining their spontaneous activity based on the analysis of their spontaneous calcium events. Note that all EG-Ins, recorded both in current- or voltage-clamp modes, already received GDPs, while $<20 \%$ of the entire GABAergic cell population was involved in GDPs $(18 \%, n=143$ neurons, 9 slices; Fig. 7A). At P7, calcium imaging was performed to detect SPA cells in hippocampal slices where E18.5 caudal ganglionic eminence-derived interneurons were specifically labeled with EGFP (Fig. 7B). We found that LG-Ins were more likely to still be producing SPAs at P7 than the overall interneuron popu- corresponds to the switch from SPA- to GDP-dominated hippocampal networks (Crépel et al., 2007). Considering all types of neurons, including glutamatergic cells, we found that a majority of SPA cells transition to GDPs over a $1 \mathrm{~d}$ period in cultures. This transition may occur at a different rate in vivo. By contrast and in agreement with the fact that interneuron genesis occurs over several days, less than half of the SPA interneurons transition to GDPs within one culture day. Moreover, since we are technically unable to follow the fate of individual cells over longer time periods, we cannot be certain that all interneurons make the SPAto-GDP transition. Indeed, given the abrupt rise in the fraction of 
GDP interneurons observed between P1 and P3, some interneuron subtypes may directly become GDP contributors. Despite this caveat, many interneurons undergo this transition, as a majority $(86 \%)$ of the newly identified GDP interneurons in slice cultures originated from cells involved in SPAs on the previous day. Furthermore, from a mechanistic standpoint, since the occurrence of GDPs actively terminates the calcium plateaus associated with SPAs (Crépel et al., 2007), persistence of these long calcium transients may ultimately trigger apoptotic programs (Golbs et al., 2011).

\section{Cellular morphophysiological correlates of SPA activity}

Significant differences in the maturation of the intrinsic electrophysiological properties were observed when comparing SPA and GDP interneurons. Independent of their morphological subtype, all SPA interneurons displayed broader and smaller action potentials, together with strongly adapting firing patterns and a depolarized $V_{\text {rest }}$. All these properties have been attributed to variations in the expression and spatial distribution of voltage-gated channels in immature cells (Moody and Bosma, 2005; Doischer et al., 2008). In addition, SPA cells display a higher averaged capacitance value, which likely reflects a larger neuronal surface (Tyzio et al., 2003), due to a larger soma size and higher membrane protrusion density. The larger soma of SPA interneurons may also account for their lower input resistance.

Membrane protrusions, and in particular filopodia, are a hallmark of developing neurons during migration and morphogenesis (Guerrier et al., 2009). However, we were not able to classify protrusions into a specific group since some resembled filopodia-like processes whereas others appeared more spine-like. Interestingly, the adult interneuron population is, with few exceptions, well known for the absence of spines in most subtypes (Freund and Buzsáki, 1996). Nonetheless, somatic protrusions in developing interneurons may, as recently proposed (Guerrier et al., 2009), contribute to the arrest of neuronal migration. The elevated intracellular chloride concentration present in immature neurons could result in osmotic pressure that acts to increase somal volume while the high intracellular calcium levels associated with SPAs could favor protrusion motility. Consistent with these ideas, both the end of interneuron migration and the emergence of SPAs are shown to depend on chloride and calcium intracellular concentrations (Crépel et al., 2007; Bortone and Polleux, 2009).

Although future studies will be required to determine the exact function of these protrusions, it may be the case that they promote the maturation of synaptic inputs onto interneurons. In general, immature synaptic inputs give rise to low-frequency and slow kinetics mPSCs that favor GABAergic inputs over glutamatergic ones (Tyzio et al., 1999; Hennou et al., 2002; Wang and
B
P3

1
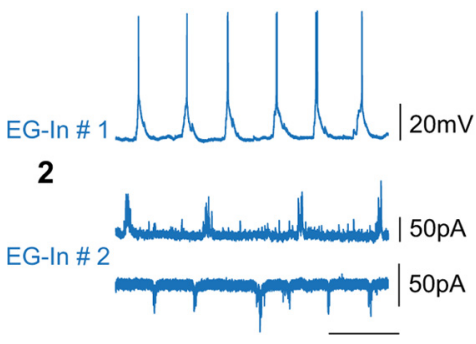

3

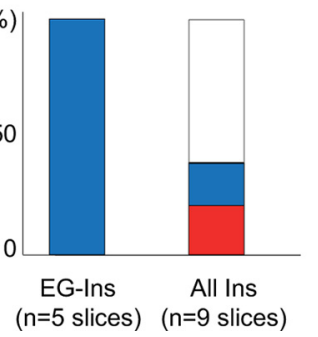

P7

1

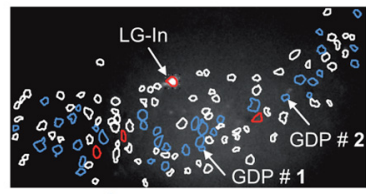

2

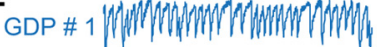

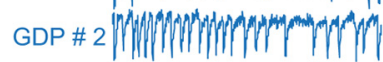

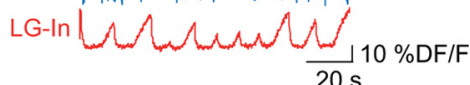

3

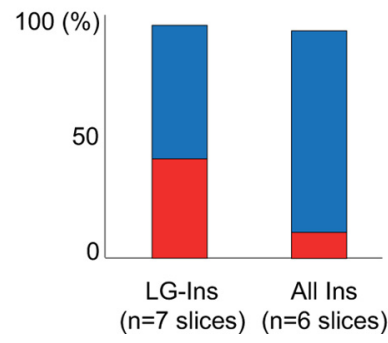

Figure 7. Interneuron involvement in SPAs or GDPs depends on birth date. A1, Current-clamp recording at resting membrane potential ( $0 \mathrm{pA}$ ) of an EG-In of the CA3 region of a P3 Mash ${ }^{\text {CreERTM }} ; R$ CE:LoxP mouse treated with tamoxifen at E11.5 (see Materials and Methods). Note the presence of GDPs. A2, Same as $\boldsymbol{A} 1$ but in voltage-clamp mode recorded at $+10 \mathrm{mV}$ (top trace) and -60 inaged in the CA1 hippocampal region of a P7 Mash $7^{\text {CreERTM }} ;$ RCE:LoxP mouse treated with tamoxifen at E18.5 (see Materials and contours, SPA-cells. B2, Traces of calcium fluorescence changes as a function of time for two representative GDP cells and for the total population of $\mathrm{LG}$-Ins from Mash $7^{\text {CreeRTM }}$;RCE:LoxP mice treated with tamoxifen at E18.5 (left) and out the total population of GAD-GFP-positive cells from GAD67 KI mice (right). Note that a large fraction of LG-Ins are still involved in SPA at a development stage (P7) dominated by GDPs.

Kriegstein, 2008). Unexpectedly, except for the decay of mEPSCs, which was faster in SPA cells, we could not find any significant difference in the frequency or kinetics of miniature and spontaneous PSCs between interneurons involved in SPAs or GDPs. This indicates that the number, distribution, and subunit composition of glutamatergic and GABAergic synaptic inputs impinging onto SPA and GDP interneurons are likely to be comparable. However, given the diversity of the GABAergic interneuron population, we cannot exclude the possibility that significant differences do exist until we are able to restrict our comparisons to interneurons of the same subtype. This remains a challenging task given the late development of classic interneuron markers and the fact that different subtypes can only be distinguished after these events have been completed.

SPA and GDP interneurons, however, differed in their PSCs amplitude distributions, with SPA interneurons possessing a higher proportion of miniature events with large amplitudes. These large amplitude PSCs raise several questions regarding both the mechanisms of their generation and their function. Given the fact that large amplitude events occur similarly for GABAergic and glutamatergic inputs, the mechanism generating them may well be postsynaptic in origin. However, we cannot exclude that SPA interneurons are not selectively innervated by 
specific presynaptic glutamatergic and GABAergic partners that, for example, share the same temporal embryonic origin (Yu et al., 2009; Deguchi et al., 2011).

We can thus conclude that the transition from a SPA to a GDP activity pattern has several morphophysiological correlates at the cellular level and inaugurates the expression of physiological diversity in GABAergic interneurons. Whether the SPA-to-GDP transition is causally mediating this morphophysiological evolution remains to be determined by specific interference experiments.

\section{Postnatal morphophysiological development of interneurons:} activity versus intrinsic programs

The previously presented results raise the question as to whether the development of cortical interneurons is more influenced by activity or by intrinsic genetic programs (Cossart, 2011; Fishell and Rudy, 2011). Previous studies suggest that the adult morphophysiological phenotype of cortical interneurons is strongly predetermined by their spatiotemporal embryonic origins (Xu et al., 2004; Butt et al., 2005; Batista-Brito and Fishell, 2009; Corbin and Butt, 2011; Tricoire et al., 2011), while early and late postnatal activities determine their final position and morphological development (De Marco García et al., 2011). One possible interpretation of our findings is that the maturation of all interneurons is dictated by their birth date and is independent of their subtype, spatial origin, or the ambient network activities they experience. This idea is, however, seemingly at odds with earlier observations noting that early- and late-born caudal ganglionic eminencederived interneurons enter the cortex and uniformly occupy the superficial laminae, independent of their birth date (Miyoshi et al., 2010). However, these latter findings pertain to the cortex rather than the hippocampus where the laminar fate of late-born neurons can be altered if network activity is disrupted during development (De Marco García et al., 2011). Perhaps the correct conclusion is that some aspects of interneuron development, such as the SPA-to-GDP transition, are genetic, while others, such as laminar position, reflect intrinsic differences within distinct subclasses. Regardless of these differences, it seems clear that genetic programs initiated at birth are crucial in dictating the time at which developing neurons synaptically integrate into functional cortical circuits (Petrovic and Hummel, 2008; Deguchi et al., 2011). In fact, the present results suggest a mechanistic basis for the previously described selective wiring between temporally matched cell populations (Deguchi et al., 2011): cells generated at the same time will likely display similar firing dynamics at critical points of development, independent of their subtype, and, as such, be predisposed to wire together. Importantly, these findings highlight that, despite the natural desire to discriminate between intrinsic genetic programs and activity, the contributions of each are inherently intertwined.

To conclude, we have shown that the SPA-to-GDP transition, which is likely to occur in several interneuron subtypes, has significant morphophysiological correlates. Since this sequence is shared across developing cortical structures (Allene and Cossart, 2010), this observation may well be generalizable. A better understanding of the parameters that govern the SPA-to-GDP switch should ultimately help us understand the etiology of the developmental brain disorders classified as interneuropathies.

\section{References}

Allene C, Cossart R (2010) Early NMDA receptor-driven waves of activity in the developing neocortex: physiological or pathological network oscillations? J Physiol 588:83-91.

Allene C, Cattani A, Ackman JB, Bonifazi P, Aniksztejn L, Ben-Ari Y, Cossart
R (2008) Sequential generation of two distinct synapse-driven network patterns in developing neocortex. J Neurosci 28:12851-12863.

Ascoli GA, Alonso-Nanclares L, Anderson SA, Barrionuevo G, BenavidesPiccione R, Burkhalter A, Buzsáki G, Cauli B, Defelipe J, Fairén A, Feldmeyer D, Fishell G, Fregnac Y, Freund TF, Gardner D, Gardner EP, Goldberg JH, Helmstaedter M, Hestrin S, Karube F, et al. (2008) Petilla terminology: nomenclature of features of GABAergic interneurons of the cerebral cortex. Nat Rev Neurosci 9:557-568.

Batista-Brito R, Fishell G (2009) The developmental integration of cortical interneurons into a functional network. Curr Top Dev Biol 87:81-118.

Ben-Ari Y, Cherubini E, Corradetti R, Gaiarsa JL (1989) Giant synaptic potentials in immature rat CA3 hippocampal neurones. J Physiol 416:303-325.

Blankenship AG, Feller MB (2010) Mechanisms underlying spontaneous patterned activity in developing neural circuits. Nat Rev Neurosci $11: 18-29$

Bonifazi P, Goldin M, Picardo MA, Jorquera I, Cattani A, Bianconi G, Represa A, Ben-Ari Y, Cossart R (2009) GABAergic hub neurons orchestrate synchrony in developing hippocampal networks. Science 326:1419-1424.

Bortone D, Polleux F (2009) KCC2 expression promotes the termination of cortical interneuron migration in a voltage-sensitive calcium-dependent manner. Neuron 62:53-71.

Buss RR, Oppenheim RW (2004) Role of programmed cell death in normal neuronal development and function. Anat Sci Int 79:191-197.

Butt SJ, Fuccillo M, Nery S, Noctor S, Kriegstein A, Corbin JG, Fishell G (2005) The temporal and spatial origins of cortical interneurons predict their physiological subtype. Neuron 48:591-604.

Corbin JG, Butt SJ (2011) Developmental mechanisms for the generation of telencephalic interneurons. Dev Neurobiol 71:710-732.

Cossart R (2011) The maturation of cortical interneuron diversity: how multiple developmental journeys shape the emergence of proper network function. Curr Opin Neurobiol 21:160-168.

Crépel V, Aronov D, Jorquera I, Represa A, Ben-Ari Y, Cossart R (2007) A parturition-associated nonsynaptic coherent activity pattern in the developing hippocampus. Neuron 54:105-120.

Deguchi Y, Donato F, Galimberti I, Cabuy E, Caroni P (2011) Temporally matched subpopulations of selectively interconnected principal neurons in the hippocampus. Nat Neurosci 14:495-504.

De Marco García NV, Karayannis T, Fishell G (2011) Neuronal activity is required for the development of specific cortical interneuron subtypes. Nature 472:351-355.

Doischer D, Hosp JA, Yanagawa Y, Obata K, Jonas P, Vida I, Bartos M (2008) Postnatal differentiation of basket cells from slow to fast signaling devices. J Neurosci 28:12956-12968.

Fishell G, Rudy B (2011) Mechanisms of inhibition within the telencephalon: "where the wild things are." Annu Rev Neurosci 34:535-567.

Freund TF, Buzsáki G (1996) Interneurons of the hippocampus. Hippocampus 6:347-470.

Garaschuk O, Hanse E, Konnerth A (1998) Developmental profile and synaptic origin of early network oscillations in the CA1 region of rat neonatal hippocampus. J Physiol 507:219-236.

Golbs A, Nimmervoll B, Sun JJ, Sava IE, Luhmann HJ (2011) Control of programmed cell death by distinct electrical activity patterns. Cereb Cortex 21:1192-1202.

Guerrier S, Coutinho-Budd J, Sassa T, Gresset A, Jordan NV, Chen K, Jin WL, Frost A, Polleux F (2009) The F-BAR domain of srGAP2 induces membrane protrusions required for neuronal migration and morphogenesis. Cell 138:990-1004.

Hennou S, Khalilov I, Diabira D, Ben-Ari Y, Gozlan H (2002) Early sequential formation of functional GABA(A) and glutamatergic synapses on CA1 interneurons of the rat foetal hippocampus. Eur J Neurosci 16:197-208.

Miyoshi G, Hjerling-Leffler J, Karayannis T, Sousa VH, Butt SJ, Battiste J, Johnson JE, Machold RP, Fishell G (2010) Genetic fate mapping reveals that the caudal ganglionic eminence produces a large and diverse population of superficial cortical interneurons. J Neurosci 30:1582-1594.

Moody WJ, Bosma MM (2005) Ion channel development, spontaneous activity, and activity-dependent development in nerve and muscle cells. Physiol Rev 85:883-941.

Okaty BW, Miller MN, Sugino K, Hempel CM, Nelson SB (2009) Transcriptional and electrophysiological maturation of neocortical fastspiking GABAergic interneurons. J Neurosci 29:7040-7052. 
Petrovic M, Hummel T (2008) Temporal identity in axonal target layer recognition. Nature 456:800-803.

Picardo MA, Guigue P, Bonifazi P, Batista-Brito R, Allene C, Ribas A, Fishell G, Baude A, Cossart R (2011) Pioneer GABA cells comprise a subpopulation of hub neurons in the developing hippocampus. Neuron 71:695-709.

Sipilä ST, Huttu K, Soltesz I, Voipio J, Kaila K (2005) Depolarizing GABA acts on intrinsically bursting pyramidal neurons to drive giant depolarizing potentials in the immature hippocampus. J Neurosci 25:5280-5289.

Stoppini L, Buchs PA, Muller D (1991) A simple method for organotypic cultures of nervous tissue. J Neurosci Methods 37:173-182.

Tamamaki N, Yanagawa Y, Tomioka R, Miyazaki J, Obata K, Kaneko T (2003) Green fluorescent protein expression and colocalization with calretinin, parvalbumin, and somatostatin in the GAD67-GFP knock-in mouse. J Comp Neurol 467:60-79.

Tricoire L, Pelkey KA, Erkkila BE, Jeffries BW, Yuan X, McBain CJ (2011) A blueprint for the spatiotemporal origins of mouse hippocampal interneuron diversity. J Neurosci 31:10948-10970.

Tyzio R, Represa A, Jorquera I, Ben-Ari Y, Gozlan H, Aniksztejn L (1999) The establishment of GABAergic and glutamatergic synapses on CA1 pyramidal neurons is sequential and correlates with the development of the apical dendrite. J Neurosci 19:10372-10382.

Tyzio R, Ivanov A, Bernard C, Holmes GL, Ben-Ari Y, Khazipov R (2003) The membrane potential of CA3 hippocampal pyramidal cells during postnatal development. J Neurophysiol 90:2964-2972.

Wang DD, Kriegstein AR (2008) GABA regulates excitatory synapse formation in the neocortex via NMDA receptor activation. J Neurosci 28:5547-5558

Xu Q, Cobos I, De La Cruz E, Rubenstein JL, Anderson SA (2004) Origins of cortical interneuron subtypes. J Neurosci 24:2612-2622.

Yu YC, Bultje RS, Wang X, Shi SH (2009) Specific synapses develop preferentially among sister excitatory neurons in the neocortex. Nature 458: 501-504. 